\title{
Aspects of SU(3) baryon extrapolation
}

\author{
R. D. Young \\ Physics Division, Argonne National Laboratory, Argonne, Illinois 60439, USA
}

\begin{abstract}
We report on a recent chiral extrapolation, based on an SU(3) framework, of octet baryon masses calculated in 2+1-flavour lattice QCD. Here we further clarify the form of the extrapolation, the estimation of the infinite-volume limit, the extracted low-energy constants and the corrections in the strange-quark mass.
\end{abstract}

Keywords: Lattice QCD, Chiral extrapolation

PACS: $12.38 . \mathrm{Gc}, 12.39$.Fe

In recent times, there has been significant advances in numerical studies of latticeregularised QCD. In particular, the past year has seen several studies of baryon systems using 3 flavours of light dynamical quarks, including Refs. [1, 2, 3, 4, 5, 6]. While the quark masses are relatively light, one still requires an extrapolation to the physical point to make comparison with observables. Here we highlight some features of a recent $\mathrm{SU}(3)$ chiral extrapolation of octet baryon masses [7].

The chiral expansion of baryon masses have been studied extensively, eg. see [8, 9]. For our formulae, we most closely follow the work of Walker-Loud [10], writing

$$
M_{B}=M^{(0)}+\delta M_{B}^{(1)}+\delta M_{B}^{(3 / 2)}+\ldots
$$

The leading term, $M^{(0)}$, denotes the degenerate mass of the baryon octet in the SU(3) chiral limit - where $m_{u}=m_{d}=m_{s}=0$. The leading corrections, as one moves away from this limit, are linear in the quark mass. Assuming SU(2) symmetry in the light quark sector, with $m_{l}=\left(m_{u}+m_{d}\right) / 2$, these leadings corrections can be written as

$$
\delta M_{B}^{(1)}=-C_{B l}^{(1)} m_{l}-C_{B s}^{(1)} m_{s},
$$

with coefficients $C_{B q}^{(1)}$ listed in Table 1 [10]. We make a convenient substitution by reexpressing the expansion in terms of $m_{\pi}$ and $m_{K}$, with $m_{l} \rightarrow m_{\pi}^{2} / 2$ and $m_{s} \rightarrow\left(m_{K}^{2}-\right.$ $\left.m_{\pi}^{2} / 2\right)$. The coefficients, $\alpha_{M}, \beta_{M}$ and $\sigma_{M}$ are then each redefined by a dimensionful scale factor, as similarly done in Ref. [1]. To the order we work in this manuscript, the use of either quark masses or meson masses squared is equivalent.

Beyond this linear order come the leading loop corrections, which have long been known to generate large corrections (at the physical quark masses), up to $\mathscr{O}(100 \%)$ of the leading terms [8, 9]. This problem in the SU(3) expansion has been overcome through introducing a finite scale into the regularization of these loop diagrams [9]. Concurrently, the introduction of a finite regularization scale was demonstrated to tame the chiral extrapolation problem for lattice QCD [11]. While these early studies required the regularization scale to be input from phenomenology, this study uses the lattice results themselves to pick this scale. 
TABLE 1. Coefficients for the leading quarkmass expansion of the octet baryons about the $\mathrm{SU}(3)$ chiral limit.

\begin{tabular}{lcc}
\hline$B$ & $C_{B l}^{(1)}$ & $C_{B s}^{(1)}$ \\
\hline$N$ & $2 \alpha_{M}+2 \beta_{M}+4 \sigma_{M}$ & $2 \sigma_{M}$ \\
$\Lambda$ & $\alpha_{M}+2 \beta_{M}+4 \sigma_{M}$ & $\alpha_{M}+2 \sigma_{M}$ \\
$\Sigma$ & $\frac{5}{3} \alpha_{M}+\frac{2}{3} \beta_{M}+4 \sigma_{M}$ & $\frac{1}{3} \alpha_{M}+\frac{4}{3} \beta_{M}+2 \sigma_{M}$ \\
$\Xi$ & $\frac{1}{3} \alpha_{M}+\frac{4}{3} \beta_{M}+4 \sigma_{M}$ & $\frac{5}{3} \alpha_{M}+\frac{2}{3} \beta_{M}+2 \sigma_{M}$ \\
\hline
\end{tabular}

TABLE 2. The relevent coefficients for the one-loop diagrams involving intermediate octet and decuplet baryons. These contributions are counted at the same chiral order in the present scheme.

\begin{tabular}{c|ccc|ccc}
\hline & \multicolumn{4}{|c|}{$\chi_{B \phi}$} & & $\chi_{T \phi}$ \\
& $\pi$ & $K$ & $\eta$ & $\pi$ & $K$ & $\eta$ \\
\hline$N$ & $\frac{3}{2}(D+F)^{2}$ & $\frac{1}{3}\left(5 D^{2}-6 D F+9 F^{2}\right)$ & $\frac{1}{6}(D-3 F)^{2}$ & $\frac{4}{3} C^{2}$ & $\frac{1}{3} C^{2}$ & 0 \\
$\Lambda$ & $2 D^{2}$ & $\frac{2}{3}\left(D^{2}+9 F^{2}\right)$ & $\frac{2}{3} D^{2}$ & $C^{2}$ & $\frac{2}{3} C^{2}$ & 0 \\
$\Sigma$ & $\frac{2}{3}\left(D^{2}+6 F^{2}\right)$ & $2\left(D^{2}+F^{2}\right)$ & $\frac{2}{3} D^{2}$ & $\frac{2}{9} C^{2}$ & $\frac{10}{9} C^{2}$ & $\frac{1}{3} C^{2}$ \\
$\Xi$ & $\frac{3}{2}(D-F)^{2}$ & $\frac{1}{3}\left(5 D^{2}+6 D F+9 F^{2}\right)$ & $\frac{1}{6}(D+3 F)^{2}$ & $\frac{1}{3} C^{2}$ & $C^{2}$ & $\frac{1}{3} C^{2}$ \\
\hline
\end{tabular}

We summarize the loop contributions by

$$
\delta M_{B}^{(3 / 2)}=-\frac{1}{16 \pi f^{2}} \sum_{\phi}\left[\chi_{B \phi} I_{R}\left(m_{\phi}, 0, \Lambda\right)+\chi_{T \phi} I_{R}\left(m_{\phi}, \delta, \Lambda\right)\right]
$$

where the loop integrals, $I$, and appropriate renormalisations, in a variety of regularisations, can be found in Ref. [12].

The relevant coefficients for these diagrams are displayed in Table 22 [10]. For this study, the baryon-baryon-meson coupling constants are fixed by phenomenology, with $D+F=g_{A}=1.27$ and from SU(6) we use $F=\frac{2}{3} D$ and $C=-2 D$. We note a similar value for $C$ can also be inferred from the decay width of the $\Delta$. We adopt a chiral perturbation theory estimate for the pion decay constant in the SU(3) chiral limit, $f=0.0871 \mathrm{GeV}$ [13]. The final phenomenological input we use is the octet-decuplet splitting, where we take the physical $N-\Delta$ splitting, $\delta=0.292 \mathrm{GeV}$. All these inputs could potentially be constrained by actual lattice simulation results, or at least in the near future. The full analysis reported in [7] allows each of the chiral axial charges to vary from these estimates by $15 \%$, and a $5 \%$ variation in $f$ - precisions which should certainly be within reach of the current or next generation of simulations.

We apply our chiral expansion to two recent 2+1-flavour calculations of the octet baryon spectrum, those by LHPC [1] and PACS-CS [2]. To determine the absolute masses of the baryons, we need to rely on the lattice scale to be fixed from an alternate source. The MILC collaboration have gone to tremendous effort to tune the lattice spacing to reproduce hyperfine splittings in $b$-quark systems [14]. For the purposes of matching, it is convenient to relate to this scale determination through the Sommer scale, using $r_{0}=0.465 \pm 0.012 \mathrm{fm}[14]$. 


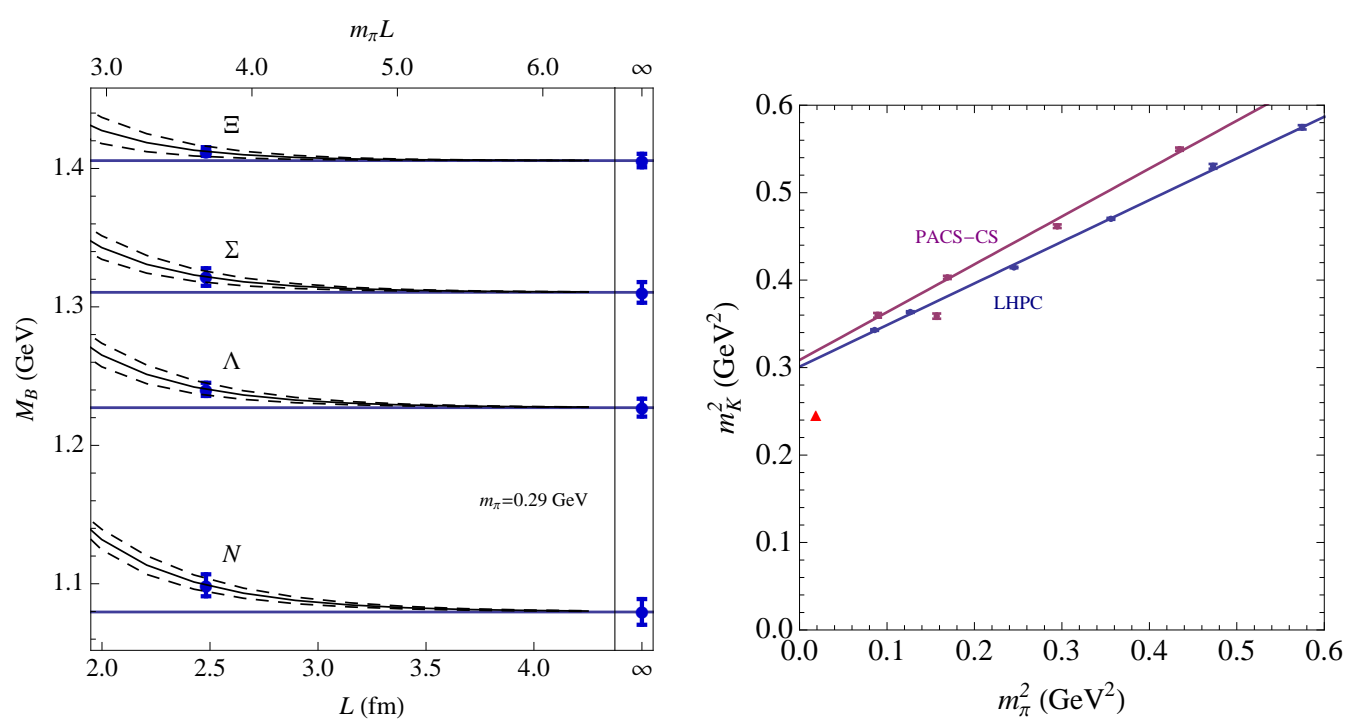

FIGURE 1. (left panel) The baryon masses are shown for the lightest pion mass of LHPC simulations. The dashed bands denote an estimate of the uncertainty in the finite-volume correction, as described in the text. The horizontal lines depict the infinite volume limit, with the combined uncertainty shown by the data point at the right edge of the graph. (right panel) Simple linear extrapolations of the kaon mass squared for the PACS-CS and LHPC results. The physical kaon mass is denoted by the triangle.

The expansion described is formulated for the continuum and infinite-volume limit. While the approach to the continuum can be improved by choice of lattice action, the finite-volume effects are dominated by infrared chiral physics. This is the same chiral physics that contributes to the chiral expansion described above, where the leading finitevolume effects can be estimated by replacing the continuum loop integrals with discrete sums over lattice momenta [15, 16, 17]. In the left panel of Figure 1, we show an example of the EFT prediction of the approach to the infinite-volume limit - a prediction that is strongly supported by numerical studies in $\mathrm{SU}(2)$ simulations [16]. Our band shows potential differences in how the ultraviolet component of the meson loops are treated, using either a finite regulator [15] or a scale-free approach [17]. The uncertainty is added in quadrature to the statistical error in the estimate of the infinite-volume limit.

We choose to remain as close to the chiral regime as possible, and fit just those results for $m_{\pi}^{2}<0.2 \mathrm{GeV}^{2}$. Independent fits to each of the LHPC and PACS-CS data sets yield excellent agreement in the low-energy constants of the fits, see Fig. 2. Further, the one additional fit parameter, given by the finite regularization scale is also in agreement between the two simulations.

This determination of the LECs and the relevant correlations lead to the accurate values for the baryon masses reported in [7]. Beyond the masses that have been extracted, the analysis demonstrates the ability to correct for the difference between the lattice and physical strange quark masses - in this case, the larger lattice strange quark masses are realised in the right panel of Fig. 1. The reliability of these corrections is demonstrated in [7], where the fit to the PACS-CS results are used to predict the baryon masses of the different strange quark mass ensemble.

Expressions for the baryon masses as a function of the meson masses (or quark masses 

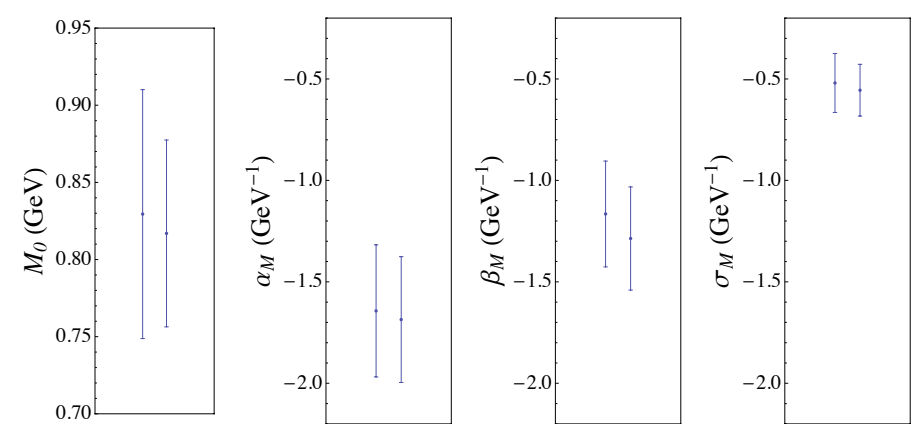

FIGURE 2. Best-fit parameters for the fits to the PACS-CS (left) and LHPC (right) lattice results.

by Gell-Mann-Oakes-Renner) have also enabled accurate determinations of the relevant sigma terms [7]. These sigma terms are of particular importance in the context of current dark matter searches [18]. Our extracted values are in satisfactory agreement with both the latest light-quark sigma term inferred from experiment [19], and other recent lattice determinations of the strange-quark sigma term [20, 21]. These "small" strangeness sigma terms are noted to be consistent with phenomenological expectations [22, 23].

I thank A. W. Thomas for collaboration on the work presented here. This work was supported by DOE contracts DE-AC02-06CH11357, under which UChicago Argonne, LLC, operates Argonne National Laboratory.

\section{REFERENCES}

1. A. Walker-Loud et al., arXiv:0806.4549 [hep-lat].

2. S. Aoki et al. [PACS-CS], arXiv:0807.1661 [hep-lat].

3. H. W. Lin et al. [HSC], Phys. Rev. D 79, 034502 (2009) [arXiv:0810.3588 [hep-lat]].

4. S. Dürr et al., Science 322, 1224 (2008) [arXiv:0906.3599 [hep-lat]].

5. S. R. Beane et al., arXiv:0903.2990 [hep-lat].

6. T. Yamazaki et al., arXiv:0904.2039 [hep-lat].

7. R. D. Young and A. W. Thomas, arXiv:0901.3310 [hep-lat].

8. B. Borasoy and U. G. Meissner, Annals Phys. 254, 192 (1997) [arXiv:hep-ph/9607432].

9. J. F. Donoghue et al., Phys. Rev. D 59, 036002 (1999) [arXiv:hep-ph/9804281].

10. A. Walker-Loud, Nucl. Phys. A 747, 476 (2005) |arXiv:hep-lat/0405007|.

11. D. B. Leinweber et al., Phys. Rev. D 61, 074502 (2000) [arXiv:hep-lat/9906027].

12. R. D. Young et al., Prog. Part. Nucl. Phys. 50, 399 (2003) |arXiv:hep-lat/0212031].

13. G. Amoros et al., Nucl. Phys. B 602, 87 (2001) [arXiv:hep-ph/0101127].

14. C. Aubin et al., Phys. Rev. D 70, 094505 (2004) [arXiv:hep-lat/0402030|.

15. R. D. Young et al., Phys. Rev. D 66, 094507 (2002) |arXiv:hep-lat/0205017|.

16. A. Ali Khan et al. [QCDSF-UKQCD], Nucl. Phys. B 689, 175 (2004) |arXiv:hep-lat/0312030|.

17. S. R. Beane, Phys. Rev. D 70, 034507 (2004) [arXiv:hep-lat/0403015].

18. J. R. Ellis, K. A. Olive and C. Savage, Phys. Rev. D 77, 065026 (2008) [arXiv:0801.3656 [hep-ph]].

19. M. M. Pavan et al., PiN Newslett. 16, 110 (2002) [arXiv:hep-ph/0111066].

20. H. Ohki et al., Phys. Rev. D 78, 054502 (2008) [arXiv:0806.4744[[hep-lat]].

21. D. Toussaint and W. Freeman [MILC Collaboration], arXiv:0905.2432 [hep-lat].

22. V. V. Flambaum et al., Phys. Rev. D 69, 115006 (2004) [arXiv:hep-ph/0402098].

23. L. Chang, I. C. Cloët, B. El-Bennich, T. Klähn and C. D. Roberts, arXiv:0906.4304 [nucl-th]. 